\title{
The Role of Quench-back in the Passive Quench Protection of Uncoupled Solenoids in Series with and without Coil Sub-division
}

\author{
X. L. Guo, M. A. Green, L. Wang, H. Wu, and H. Pan
}

\begin{abstract}
This paper is the final paper in a series of papers that discusses passive quench protection for high inductance solenoid magnets [1], [2]. This report describes how passive quench protection system may be applied to superconducting magnets that are connected in series but not inductively coupled. Previous papers have discussed the role of magnet sub-division and quench back from a conductive mandrel in reducing the hot-spot temperature and the peak coil voltages to ground. When magnets are connected in series, quench-back from a conductive mandrel can cause other magnets in a string to quench even without inductive coupling between magnets. The magnet mandrels must be well coupled to the magnet circuit that is being quenched. When magnet circuit sub-division is employed to reduce the voltages-to-ground within magnets, the resistance across the subdivision becomes the most important factor in the successful quenching of the magnet string.
\end{abstract}

Index Terms-Quench-back, Sub-division quench protection

\section{INTRODUCTION}

Q uench-back is a widely used passive quench protection method that enhances normal zone propagation by using a coupled secondary circuit. In one magnet quench-back will initiate further quenching, thus effectively increasing quench propagation velocity. It is particularly effective in a situation where the normal zone hits boundaries early in the quench process and thereafter spreads very slowly in the other dimensions [3-5]. When magnets are connected in series, quench-back can potentially cause the other magnets to quench even if they are not inductively coupled [6] [7].

Sub-division provides an alternative path for some of the current by connecting shunt resistors across sections of the magnet, and the shunt resistor can absorb some of the stored energy in the coil. The magnet hot spot temperature can be reduced by this technology. The shunt resistors across the sub-division can balance the overvoltage in the coil, so the voltage to ground in the coil can be limited and reduced [8].

Manuscript received 1 August 2010. This work is supported by the Office of Science of the US Department of Energy under DOE contract DE-AC-02-05CH11231.

X. L. Guo is with Jiangsu University, Zhenjiang 212013, China. L. Wang is with Shanghai Institute of Applied Physics, Shanghai 201204, China. H. $\mathrm{Wu}$ is with Institute of Cryogenics and Superconductivity Technology, HIT, Harbin 150001, China. H. Pan is with the Institute of High Energy Physics, Beijing, 10049, China

M. A. Green is with Lawrence Berkeley National Laboratory, Berkeley, CA, 94720 USA (e-mail: magreen@lbl.gov).
Previous papers have discussed the quench process, the role of quench-back from the mandrel, and magnet sub-division in reducing the hot-spot temperature and the peak coil voltages to ground [1], [2], and [8]. This paper discusses the role of quench-back in the passive quench protection of uncoupled solenoids in series with and without coil sub-division.

\section{Quench Protection of A MiCE Focusing Magnet}

The solenoid discussed in this paper is the MICE focusing magnet [9] [10]. The focusing magnet focuses the muon beam into a liquid hydrogen absorber in the magnet warm bore. Fig. 1 shows a cross-section of half of the MICE focusing magnet. Each focusing magnet consists of two coils $\mathrm{C} 1$ and $\mathrm{C} 2$ wound on a 6061-T6-Al mandrel. The two coils are connected in series. The coil inner radius is $263 \mathrm{~mm}$; the coil thickness is $84 \mathrm{~mm}$; and the coil length is $210 \mathrm{~mm}$. The separation between coils $\mathrm{C} 1$ and $\mathrm{C} 2$ is $200 \mathrm{~mm}$.

The conductor used in the simulation is $\mathrm{Nb}-\mathrm{Ti}$ in copper with a $\mathrm{Cu}$ to $\mathrm{S} / \mathrm{C}$ ratio of 4 . The copper RRR is 70 . The insulated conductor dimensions are 1.65 by $1.00 \mathrm{~mm}$. The conductor has 220 filaments with a twist pitch of $\sim 19 \mathrm{~mm}$.

The focusing magnets may be run in two modes in the MICE experiment. The coils $\mathrm{C} 1$ and $\mathrm{C} 2$ can be run at the same polarity (the non-flip mode) or at opposite polarity (the flip mode). Table. 1 shows the parameters for the focusing magnet in both modes. The circuit self-inductance depends on whether the magnets in the circuit run in the flip mode or the non-flip mode or a mixture of both modes. The coils within any given focusing magnet are inductively coupled to each other and to their mandrel. The coupling between one focusing magnet and another is poor, because the focusing magnet centers are separated by $2.75 \mathrm{~m}$.
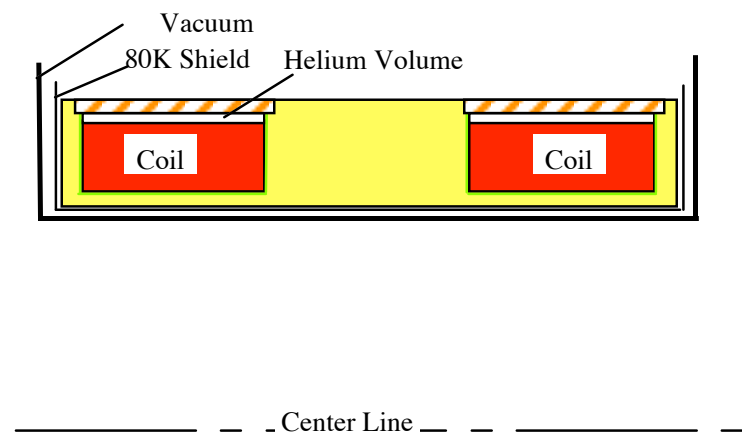

Fig.1. A Schematic Cross-section of the MICE Focusing Magnet Coils 
TABle I. MICE Focusing MAgnet PARAMETERS

\begin{tabular}{lcc}
\multicolumn{1}{c}{ Parameter } & Value \\
\hline \hline Number of Coils & 2 & \\
Coil Length (mm) & 210 & \\
Coil Inner Radius (mm) & 263 & \\
Coil Thickness (mm) & \multicolumn{2}{c}{84} \\
Coil Separation (mm) & \multicolumn{2}{c}{200} \\
Number Turns per Layer & \multicolumn{2}{c}{127} \\
Number Layers per Coil & \multicolumn{2}{c}{76} \\
Operation Mode & Flip & Non-flip \\
Design Current $\mathrm{I}_{\mathrm{D}}(\mathrm{A})$ & 250 & \multicolumn{2}{c}{195} \\
Magnet Self Inductance (H) & 98.6 & \multicolumn{2}{c}{137.4} \\
Stored Energy at $\mathrm{I}_{\mathrm{D}}(\mathrm{MJ})$ & 3.08 & 2.61 \\
\hline \hline
\end{tabular}

Fig. 2 shows a design case for the quench protection circuit for the MICE focusing magnets. The three focusing magnets are connected in series and powered by a power supply that delivers $300 \mathrm{~A}$ at $10 \mathrm{~V}$. The focusing magnets shown in Fig.2 show the quench protection diodes inside of the magnet cryostat, which protects the magnet in the event a lead fails.

When considering a single focusing magnet, it is desirable a quench in one coil can cause the other coil in the magnet to quench using quench-back from the conductive mandrel.

When three focusing magnets are connected in series, the quench of one magnet is unlikely to drive the second magnet to quench through the $\mathrm{AC}$ loss in the winding or by driving the second magnet to its critical current, because there is very little coupling between focusing magnets. The quench of one magnet may put enough energy into the mandrel of the second magnet and cause it to quench using quench-back.

Quench-back can spread the quench from a magnet to another magnet and reduce the hot spot temperature in the coil. Sub-division of the focusing magnet is used to reduce the voltage to ground and further reduce the magnet hot spot temperature.

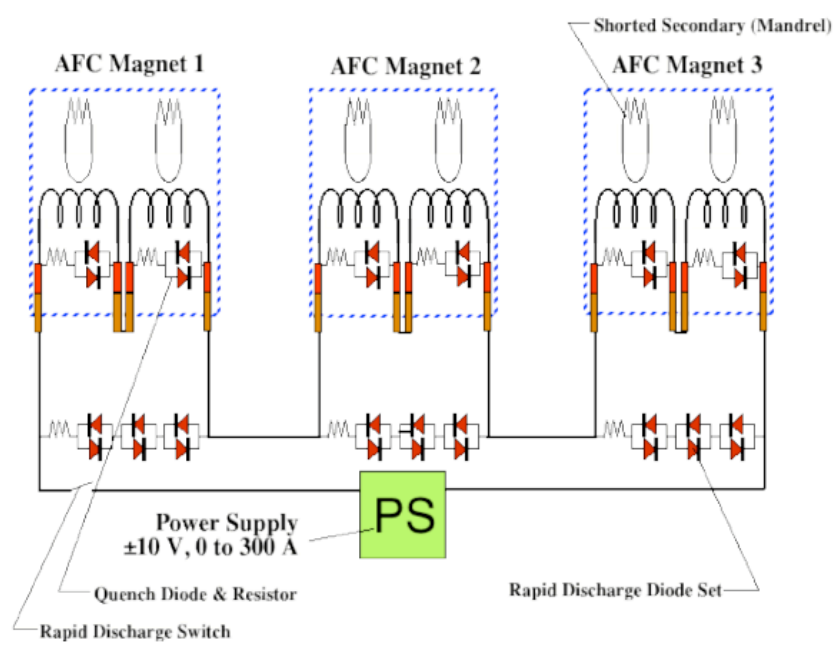

Fig.2. Quench protection circuit for the MICE focusing magnets

\section{Quench Results of A Single Focusing Magnet}

\section{A. Computation Model description}

A semi-empirical quench model considering both the sub-division and quench-back was developed to study the quench process of the MICE solenoids [1]. For the focusing magnets, the quench is initiated at the high field point in coil $\mathrm{C} 1$ and starts to expand in the three directions with velocities $v_{\phi}$ (propagation along the winding), $v_{\mathrm{r}}$ (radial propagation) and $v_{z}$ (axial propagation). In the model, average constant quench propagation velocities in three directions are used. The calculated propagation velocities are based on a correlation of experimental values as a function of average coil field and the conductor current density [7] [11]. For the focusing magnet in the flip mode, $v_{\phi}$ is $4.3 \mathrm{~ms}^{-1}, v_{\mathrm{r}}$ is $0.06 \mathrm{~ms}^{-1}$ and $v_{\mathrm{z}}$ is $0.10 \mathrm{~ms}^{-1}$.

The normal zone shape is assumed to be an ellipsoid. The calculation proceeds in time steps of $\Delta t$. At each step another layer is added to the normal zone surface. After a time step the current has decayed. The calculation proceeds until the each coil current is less than 1 percent of the starting current [12].

For the quench-back modeling, the key parameter is the time when the new normal zone induced by the heat from the mandrel. This time is the sum of two time periods. The first time period is the time for the mandrel to heats up to $\sim 10 \mathrm{~K}$. This time period is a function of the mandrel resistivity and volume specific heat [4]. The second time period is the time needed for the heat to flow across the mandrel insulation. This time is usually much shorter than the first time period [4].

\section{B. The role of Quench-back in One Focusing Magnet}

A low mandrel resistivity and specific heat leads to a faster mandrel temperature rise to a specific temperature $(\sim 10 \mathrm{~K})$, so that quench back from the mandrel will occur earlier. Mandrels of 6061-T6-Al and 304-stainless steel were used to explore the role mandrel material on quench-back [2]. The focusing magnet was run in the flip mode at $250 \mathrm{~A}$. Each coil has one sub-division (see Fig. 2). The resistance across each section is $0.02 \Omega$. Quench is initiated in Coil C1. Coil C2 eventually quenches through quench-back.

Fig. 3 shows the coil current versus time for different mandrel materials. With a 6061-T6 mandrel, quench back quenches coil $\mathrm{C} 2$ after about $2.5 \mathrm{~s}$. With a $304 \mathrm{SS}$ mandrel, the current in coil $\mathrm{C} 2$ decays slowly with no quench-back.

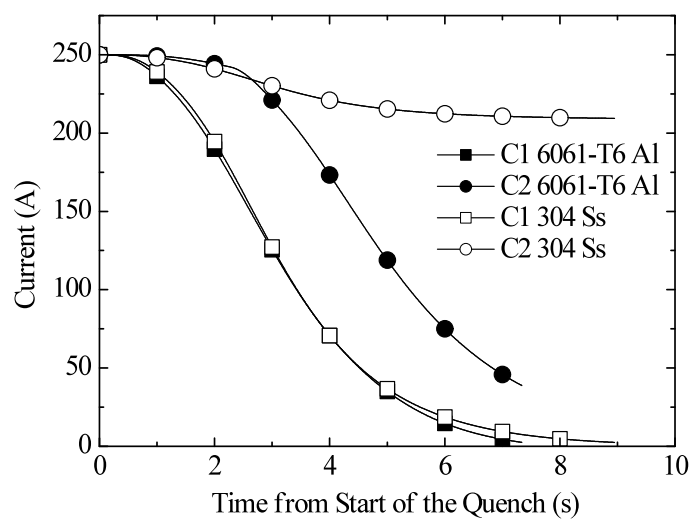

Fig. 3. Currents in coils with different mandrel materials 
Fig. 4 shows that the hot spot temperature varies with the mandrel material. With 6061-T6 Al and $304 \mathrm{SS}$, the hot spot temperature is $109 \mathrm{~K}$ and $112 \mathrm{~K}$ respectively. With $304 \mathrm{SS}$, there is no quench-back in the time shown in Figs. 3 and 4.

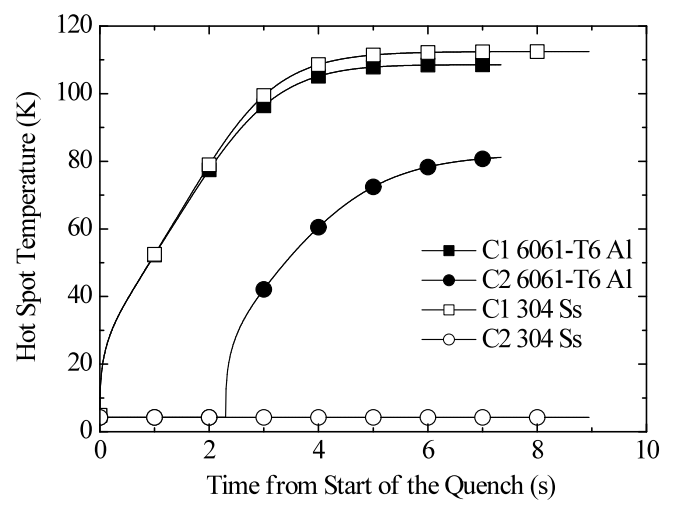

Fig. 4. Hot spot temperature in coils with different mandrel materials

Fig. 5 shows the peak voltage to ground with different mandrel materials. With 6061-T6 Al and $304 \mathrm{SS}$, the peak voltage to ground is $3689 \mathrm{~V}$ and $4004 \mathrm{~V}$ respectively. The voltage-to-ground used in the model is the resistive voltage across the normal zone. This voltage is the product of the current in the sub-division and normal zone resistance in the sub-division. Quench back can cause a small increase in the voltage to ground in the coil, because the coil section resistance grows more rapidly. If the resistivity of the mandrel is lower (say 1100-O aluminum) the peak voltage will be lower, because the mandrel absorbs more magnetic energy [2].

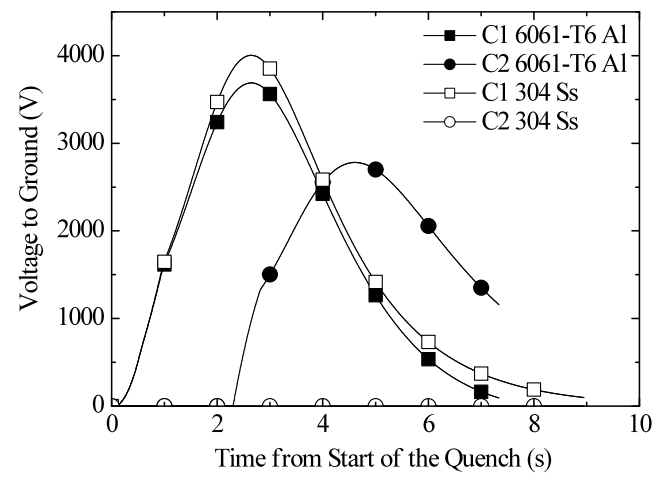

Fig. 5. Peak voltage to ground in coils with different mandrel materials

\section{The Role of Coil Subdivision in One Focusing Magnet}

The role of sub-division in the quench protection was studied for a single focusing magnet. Each coil is subdivided into one or two sections. A cold diode and $0.02 \Omega$ shunt is put across each sub-division. The cases that were run are for the focusing magnet in flip mode. The mandrel is assumed to be 6061-T6-aluminum.

Fig. 6 shows that the magnet hot spot temperature varies with the number of sub-divisions. With 1 sub-division and 2 sub-divisions, the hot spot temperature is $109 \mathrm{~K}$ and $99 \mathrm{~K}$ respectively. A larger number of sub-divisions results in a somewhat lower hot spot temperature. A quench was initiated in the second coil about $2.2 \mathrm{~s}$ after the first coil quenched.

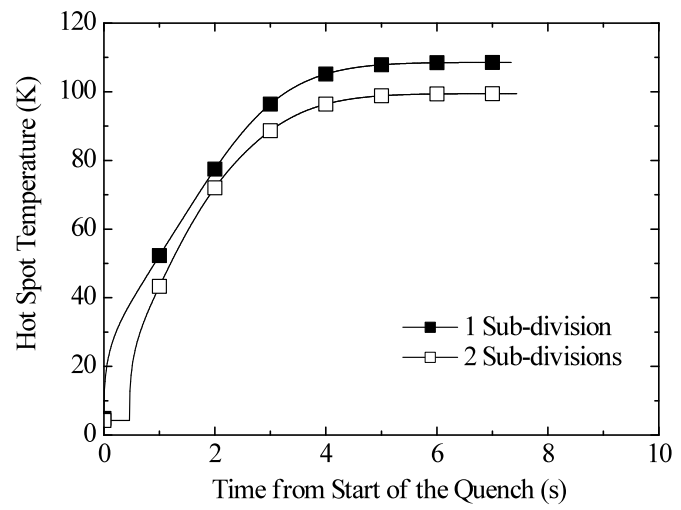

Fig. 6. Hot spot temperature in a magnet with 1 and 2 Sub-divisions per coil

Fig. 7 shows the peak voltage to ground as a function of the number of coil sub-divisions. With one coil sub-division and two coil sub-divisions, the peak voltage to ground is $3689 \mathrm{~V}$ and $1924 \mathrm{~V}$ respectively. It is clear that sub-dividing the focusing magnet coils is a desirable thing to do from the standpoint of the peak voltage to ground.

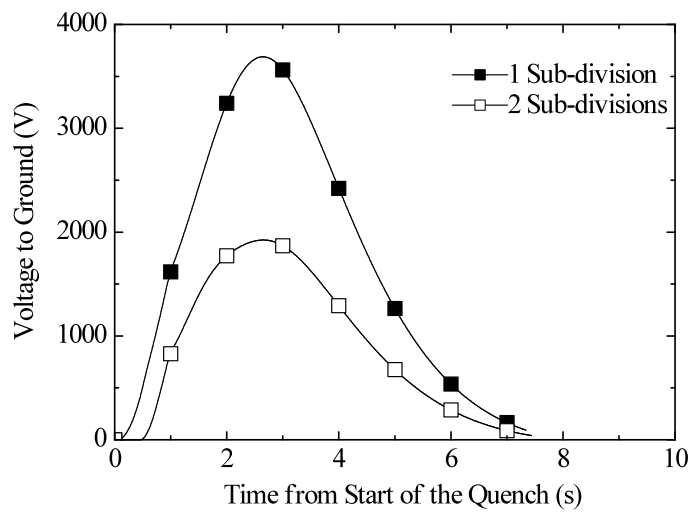

Fig. 7. Peak voltage-to-ground in magnet with 1 and 2 sub-divisions

\section{The Role of Shunt Resistance in One Focusing Magnet}

The role of shunt resistance in the quench protection was studied for a single focusing magnet and for a string of focusing magnets. Each coil has one set of back-to-back diodes and a resistor. Resistances of $0.02 \Omega$ and $2.00 \Omega$ were put across each section. The focusing magnet is assumed to be running at flip mode, at a current of $250 \mathrm{~A}$. The magnet mandrel is assumed to be 6061-T6-aluminum. Quench back causes the second coil to quench about $2.2 \mathrm{~s}$ after the first coil quenches. The peak voltage to ground is in the first coil.

Fig. 8 shows how the hot spot temperature varies with shunt resistance. With $0.02 \Omega$ and $2.00 \Omega$ resistance, the hot spot temperature is $109 \mathrm{~K}$ and $100 \mathrm{~K}$ respectively. Fig. 9 shows the peak voltage to ground with different shunt resistances. With $0.02 \Omega$ and $2.00 \Omega$ resistance, the peak voltage to ground is $3689 \mathrm{~V}$ and $2966 \mathrm{~V}$ respectively. The larger shunt resistance reduced the hot-spot temperature and the peak voltage. 


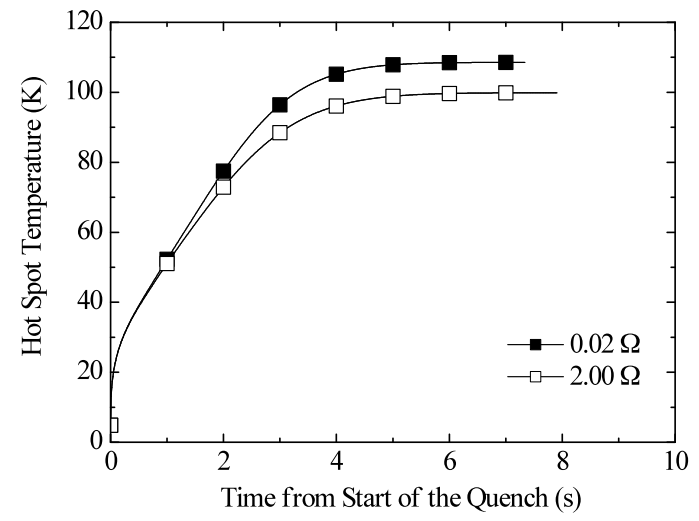

Fig. 8. Hot-spot temperature in the magnets with different shunt resistances

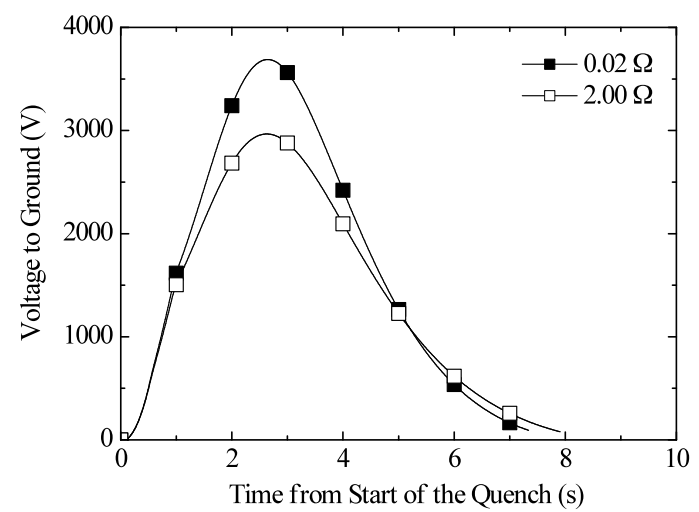

Fig. 9. Peak voltage-to-ground in magnets with different shunt resistances

With 2-ohm shunt resistance is the second coil quenches faster because of quench back. The added resistance across the coil decreases the quench-back time, because there is more current in the mandrel to drive the quench-back process.

\section{E. Summary of Single Magnet Results}

Quenching of the second coil of a single focusing magnet is driven by quench-back from the mandrel upon which the coil is wound. A 6061-T6-aluminum mandrel has a low enough resistivity and specific heat to cause quench back of the second coil within 2.2 seconds. When the first coil quenches, the diodes across both coils will fire. As a result, the second coil is decoupled from the first coil. At first the second coil will decay with the $\mathrm{L} / \mathrm{R}$ time constant for that coil and resistance combination. Then quench-back occurs. With a stainless steel mandrel quench-back does not occur.

Even without quench-back, the second coil will eventually turn normal because there is heat conduction from the quenched first coil through the mandrel material. The time before the second coil quenches is difficult to predict. One can speed up the quench process in the second coil by using the heat from the resistor across the first coil to quench the second coil and vice versa. In order to do this, there must be full ground plane insulation between the coil and the quench resistor. One must worry about insulation failure. In the view of the authors of this paper, quench-back is necessary for passive quench protection of the focusing magnet.

\section{Quench Results For FocUsing MAGNeTS IN SERIES}

The quenching of one magnet can cause a second magnet to quench through quench-back. Whether quenching one magnet can cause a quench in another magnet depends on a number of factors. They are; 1) the resistance of the resistor in series with the diodes across the coil, 2) the coupling coefficient between the mandrel inducing the quench and the coil that is quenching, 3) the temperature margin of magnet being quenched through quench back, and 4) the thermal time constant for the heat entering the coil from the mandrel [7].

The coupling coefficient and the temperature margins are important factors in determining whether a quench in one magnet will cause an adjacent magnet to quench through quench back from its mandrel. When coupling between the magnets is poor, the resistance across the coils becomes the critical parameter that causes quench-back in the second magnet. In general, thermal time constant is small compared to the time for the mandrel to heat up so say $10 \mathrm{~K}$.

TABLE 2 shows the coupling coefficient for focusing magnet with its own mandrel and with a second focusing magnet mandrel in the flip and non-flip modes.

\begin{tabular}{lcc}
$\begin{array}{c}\text { TABLE II. CALCULATED COUPLING COEFFICIENTS BETWEEN } \\
\text { THE FOCUSING MAGNETS AND THEIR MANDRELS }\end{array}$ \\
\hline \hline \multicolumn{4}{c}{ Secondary Circuit } & \multicolumn{2}{c}{ Flip } & Non-Flip \\
& 0.00000 & 0.73576 \\
To its Own Mandrel & 0.00053 & 0.00206 \\
To Nearest Focusing Mandrel & & \\
\hline \hline
\end{tabular}

The coupling coefficient between the focusing coils and their own mandrel in the flip mode is a special case because the coils operate at opposite polarity, so the sum of the terms in the inductance matrix is zero. The part of mandrel close to a coil is in fact well coupled to its own coil [7]. To estimate the coupling coefficient one has to split the mandrel with a plane in the middle of the section between the coils. Currents induced in the mandrel will be zero at the plane between the two coils. The currents in the mandrel on the two sides of the dividing plane have opposite polarity. During the quench of a single magnet the location of the zero current plane shifts because the current in one coil decays faster than the current in the second coil. When the focusing magnet is in the flip mode, the region next to the coils is hotter than the part of the mandrel between the coils. The effective coupling of a coil to the mandrel close to the coil is the same in both modes.

The coupling coefficient between a coil and a second magnet mandrel the flip mode is smaller than that in the non-flip mode. At a distance the two coils in the flip mode appear like a solenoid doublet. The spacing between two focusing magnet centers is ten times the coil spacing.

\section{A. Effect of Operation in the Flip and the Non-flip Modes}

Fig. 10 shows that the current in the coils $\mathrm{C} 1$ of both magnets in different operating modes. In both modes a quench in coil $\mathrm{C} 1$ does not induce a quench in the second magnet. The distance between the quenching magnet and the 
second magnet is too large. The final temperature of the mandrel of the second magnet in flip mode and non-flip mode is about $4.25 \mathrm{~K}$ and $4.32 \mathrm{~K}$ respectively. A low mandrel temperature in the second mandrel can't induce quench in the second magnet. The current in the flip mode decays faster than the current in the non-flip mode, because the current density is higher. In both cases, the resistance in series with the diodes across the coils is 0.02 ohms.

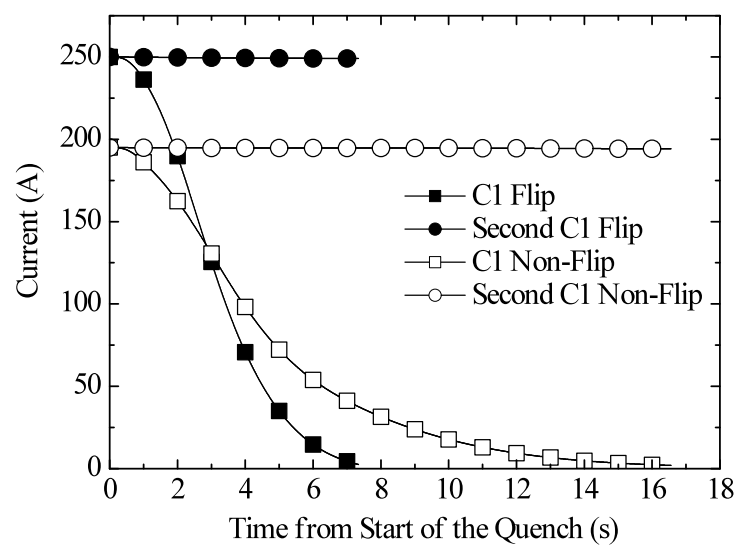

Fig. 10. The currents in coil 1 of both magnets as a function of time.

Fig. 11 shows that the hot spot temperature in the coils $\mathrm{C} 1$ and $\mathrm{C} 2$ of the quenched magnet varies for the two modes. In the flip mode and non-flip mode, the hot spot temperature is $109 \mathrm{~K}$ and $84 \mathrm{~K}$ respectively. The higher stored energy in flip mode results in a higher hot-spot temperature. From Fig. 11, one can see that the quench back time is longer for the magnet in the non-flip mode, because the coil current is lower.

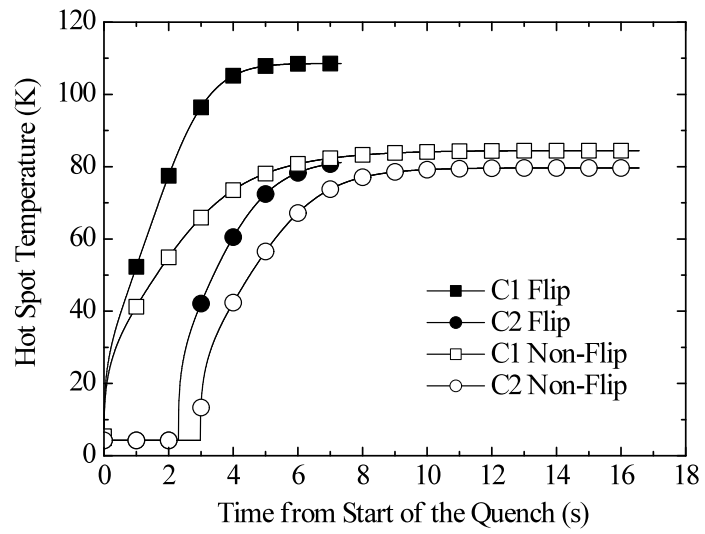

Fig.11. The hot spot temperature in the coils of the quenched magnet while operating in the flip and non-flip mode. The hot-spot temperature in the second magnet is low, because the magnet hasn't quenched.

Fig. 12 shows the peak voltage to ground in coils $\mathrm{C} 1$ and $\mathrm{C} 2$ of the quenched magnet for the two modes. In the flip mode and the non-flip mode, the peak voltage to ground is $3689 \mathrm{~V}$ and $2491 \mathrm{~V}$ respectively. The higher coil current density and stored energy while the magnet operates in flip mode results in a higher peak voltage to ground.

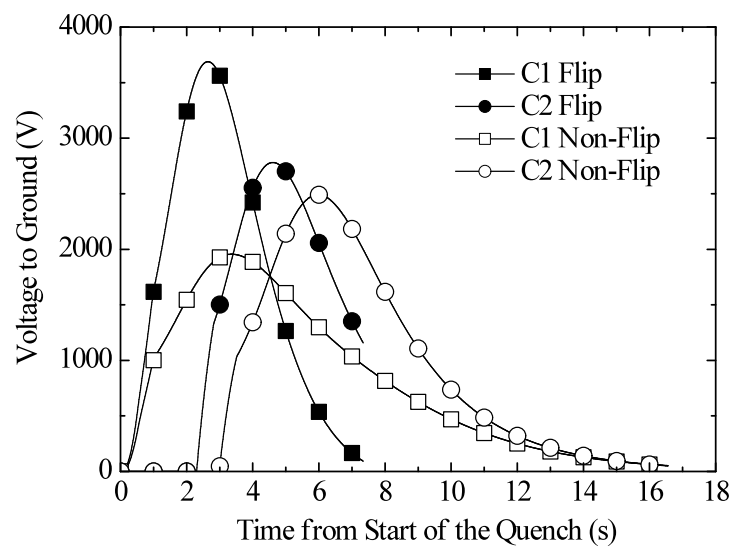

Fig. 12. The Peak voltage to ground in the coils of the quenched Magnet as a function of the focusing magnet operating Mode (In the second magnet the peak voltage are determined by the shunt resistance and as such are low.)

When the resistances across the sub-divisions are low, the focusing magnets must not be connected in series. The second and third magnets in a string of magnets may eventually quench because of heat generated in the diodes and resistors, but this effect is not predictable. As a result, there could be damage to the magnets that quench late.

\section{B. The Role of Resistance Across the Coils on Quench-back}

A resistance of 0.02 ohms across coil the sub-divisions in the focusing magnet will not induce quench-back in the other magnets in the magnet string. Increasing the resistance across the coil sub-divisions of all of the magnets to 2 ohms will cause the other magnets in the string to quench when the first magnet quenches. Fig. 13 shows the current versus time for coils $\mathrm{C} 1$ and $\mathrm{C} 2$ of the quenched magnet and a second magnet. Note the current profiles for both coils in the second magnet are the same, whereas the current profiles are different for the magnet coils where the quench originated. The hot-spot temperature versus time for all four coils is shown in Fig. 14. The voltage-to-ground for all four coils is shown in Fig. 15. The case shown in figs. 13,14 , and 15 is a flip mode case.

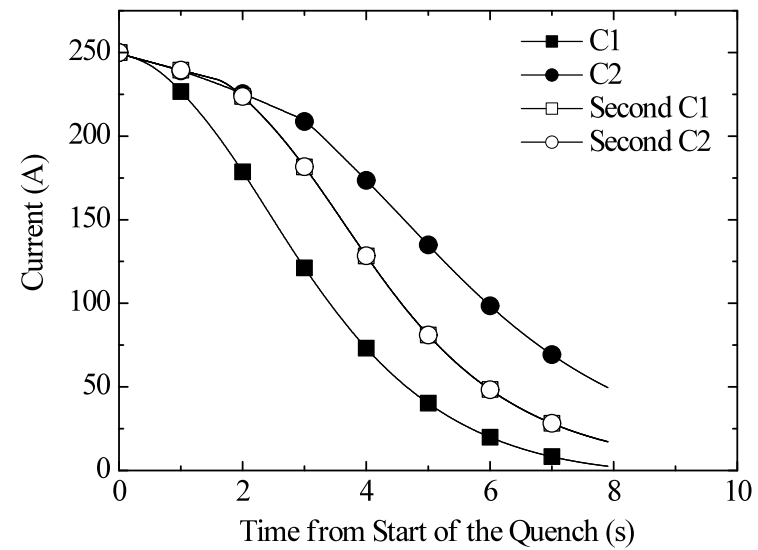

Fig. 13. The currents in both coils in both Magnets as a function of time when the resistance across the sub-division is 2 ohms. 


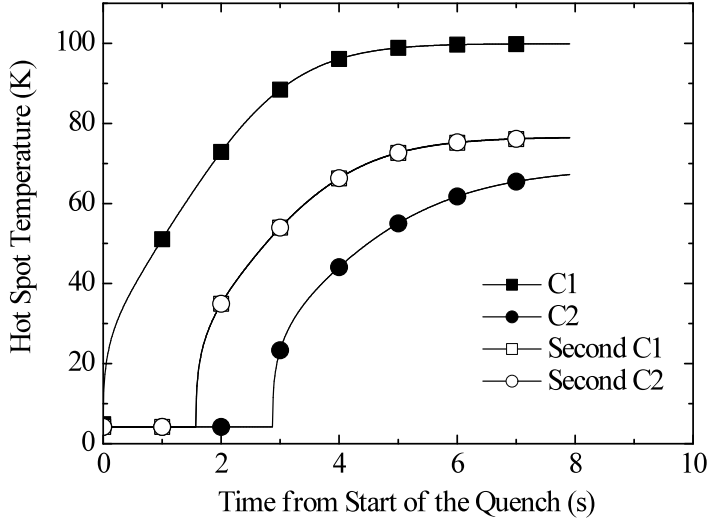

Fig. 14. The hot-spot temperature in both coils in both magnets as a function of time when the resistance across the Sub-division is $2 \mathrm{ohms}$.

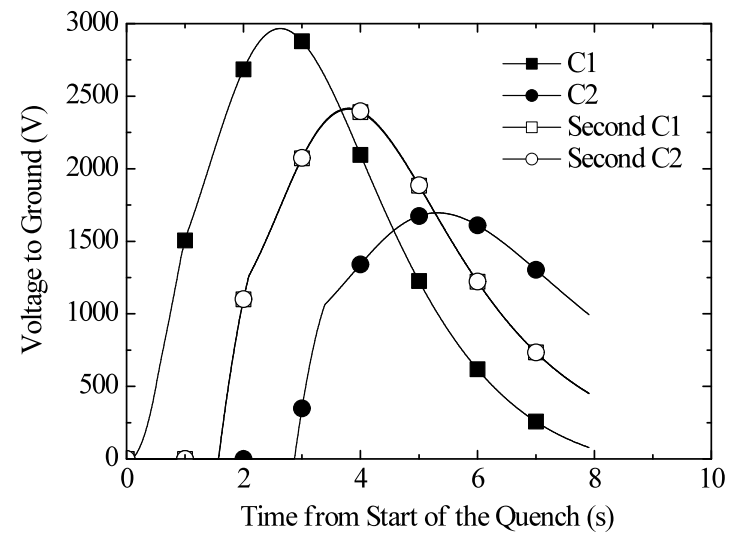

Fig. 15. The peak voltage-to-ground in both coils in both magnets as a function of time when the resistance across the sub-division is 2 ohms.

From Figs. 14 and 15, it is clear that both coils in the second magnet quench through quench-back. The quench back time for the second magnet with a $2 \mathrm{ohm}$ resistor across the sub-division is $\sim 1.6 \mathrm{~s}$. The quench back time for the magnet where the quench originated is $\sim 2.8 \mathrm{~s}$. This results in a higher hot-spot temperature and voltage-to-ground in the coil of the focusing-magnet where the quench originated.

Increasing the resistance across the sub-division to $5 \mathrm{ohms}$ reduces the hot-spot temperature to $85 \mathrm{~K}$ versus $109 \mathrm{~K}$ and $100 \mathrm{~K}$ for the cases with $0.02 \mathrm{ohms}$ and $2 \mathrm{ohms}$ respectively. Increasing the resistance across the sub-division to $5 \mathrm{ohms}$ reduces the peak voltage-to-ground to $2000 \mathrm{~V}$ versus $3689 \mathrm{~V}$ and $2950 \mathrm{~V}$ for the cases with 0.02 ohms and 2 ohms respectively.

The resistor across the coils must be non-inductive and it must be designed to absorb energy from the magnet. The resistor must have enough mass to prevent it from overheating during the quench. The quench protection resistor mass should be about $2 \mathrm{~kg}$ per ohm of resistance.

\section{CONCLUding COMMENTS}

A single focusing coil can be quench protected using a combination of the diodes and resistors across the coils and by quench-back from the 6061-T6-aluminum mandrel. The second coil in the focusing magnet will not go normal through quench back if the mandrel is made from 304-stainless steel. We recommend that each coil in the focusing magnet be sub-divided into two parts, with diodes and a resistor across each part. Increasing the resistance across the coils reduces both the hot-spot temperature and the peak voltage-to-ground. The resistor must have enough mass to prevent over heating.

The focusing magnets must not be connected in series unless the resistance across the coil sub-division is 2 ohms or higher. When the diodes fire in the magnets that are in series with the quenching magnet, the voltage across the resistors must be high enough to induce quench-back in the other magnets in the string [7]. The resistor across the coil sub-division must be non-inductive and it must have enough mass to absorb the energy from the current flowing through the resistor as it quenches. The resistor mass should be about $2 \mathrm{~kg}$ per ohm of resistance in the shunt resistor.

Having the power supply connected has little effect on the quench process, but it must be disconnected after the quench is finished to prevent the protection resistors from overheating.

\section{REFERENCES}

[1] X.L. Guo, F. Y. Xu, L. Wang, et al, "Quench Protection for the MICE Cooling Channel Coupling Magnets," IEEE Transaction on Applied Superconductivity 19, No. 3, p 1360, (2009).

[2] X. L. Guo, M. A. Green, L. Wang et al, "The Role of Quench-back in the Passive Quench Protection of Long Solenoids with Coil Sub-division," IEEE Transactions on Applied Superconductivity 20, No 3, p 2035 (2010).

[3] M. N. Wilson, Superconducting Magnets, Oxford University Press, Oxford, 1983. pp 209-232.

[4] M. A. Green, "Quench Back in Thin Superconducting Solenoid Magnets". Cryogenics 24, pp 3-10, 1984.

[5] M. A. Green, "The Role of Quench Back in Quench Protection of a Thin Superconducting Solenoid". Cryogenics 24, pp 659-668, 1984.

[6] M.A. Green, H. Witte, B.P. Strauss. "The Inductive Coupling of the Magnets in MICE and its Effect on Quench Protection," IEEE Transactions on Applied Superconductivity 16, No. 2, p 1304 (2006).

[7] M. A. Green and H. Witte, "Quench Protection and Power Supply Requirements for the MICE Focusing and Coupling Magnets," MICE Note 114, http://www.mice.iit.edu, LBNL-57580, May 2005.

[8] M. A. Green, L. Wang, X. L. Guo, et al, "Quench Protection for the MICE Cooling Channel Coupling Magnet," MICE Note 193, LBNL-63698, http://www.mice.iit.edu, November 2007.

[9] G. Gregoire, G. Ryckewaert, L. Chevalier, et al, "MICE and International Muon Ionization Cooling Experiment Technical Reference Document", [on line] http://www.mice.iit.edu, 2001.

[10] S. Q. Yang, M. A. Green, G Barr, et al, "The Mechanical and Thermal Design for the MICE Focusing Solenoid Magnet System," IEEE Transactions on Applied Superconductivity 15 No. 3, p 1269, (2005).

[11] P. H. Eberhard et al, "The measurement and Theoretical Calculation of Quench Velocities in Large Fully Impregnated Superconducting Coil, “ IEEE Transactions on Magnetics MAG-17, No. 5, p 1803, (1981)

[12] C. H. Joshi and Y. Iwasa, "Prediction of the Current Decay and Terminal Voltages in Adiabatic Superconducting Magnets," Cryogenics 29, pp 157-167 (1988) 


\section{DISCLAIMER}

This document was prepared as an account of work sponsored by the United States Government. While this document is believed to contain correct information, neither the United States Government nor any agency thereof, nor The Regents of the University of California, nor any of their employees, makes any warranty, express or implied, or assumes any legal responsibility for the accuracy, completeness, or usefulness of any information, apparatus, product, or process disclosed, or represents that its use would not infringe privately owned rights. Reference herein to any specific commercial product, process, or service by its trade name, trademark, manufacturer, or otherwise, does not necessarily constitute or imply its endorsement, recommendation, or favoring by the United States Government or any agency thereof, or The Regents of the University of California. The views and opinions of authors expressed herein do not necessarily state or reflect those of the United States Government or any agency thereof, or The Regents of the University of California. 